\title{
Sistemas de control y automatización en el aula: Estrategia didáctica en la enseñanza de las ciencias. Innovación, tecnología y creatividad.
}

\section{Control and automation systems in the classroom: Didactic strategy in the teaching of science. Innovation, technology and creativity.}

\section{Edil Melo Jaimes', Daissy M. Payares Benítez², Silvia M. Baldiris Navarro³}

${ }^{1}$ Mg., Docente, Área de Ciencias Naturales y Educación Ambiental, CSI INEDSOR, Institución Educativa Soledad Román de Núñez, Cra. 57 \#30D-47 Escallón Villa, Cartagena, Colombia.

edilmjaimess@gmail.com

${ }^{2}$ Mg., Docente, Área de Tecnología e Informática, ECO INEDSOR, Institución Educativa Soledad Román de Núñez, Cra. 57 \#30D-47 Escallón Villa, Cartagena, Colombia.

profe.daissy@gmail.com

${ }^{3}$ Ph.D. Docente, Fundación Universitaria Tecnológico Comfenalco, (Cartagena, Colombia) y Universidad Internacional de la Rioja (Logroño, España), Cartagena, Colombia

sbaldiris@gmail.com

Recibido: 15/ago/2019 - Revisado: 30/sep/2019

Aceptado: 30/oct/2019 - Publicado: 30/jun/2019

Resumen Los entornos de desarrollo - IDE - y sus aplicaciones en experimentos de las ciencias naturales basados en la medición, han tenido gran acogida por la eficiencia y facilidad en la comprobación de teorías científicas; al tener más interacción con los sistemas de control y automatización como estrategia pedagógica en el aula, en instituciones educativas de secundaria, ha permitido en los estudiantes, espec íficamente de los grados noveno a undécimo, lograr mayor confianza, motivación, trabajo colaborativo y uso de las TIC para el aprendizaje de las ciencias naturales, usando el aprendizaje significativo para el diseño y construcción de un invernadero automatizado para la germinación de mangle negro (Avicennia germinans), obteniendo un crecimiento óptimo y conservación de las condiciones ambientales ideales desde la programación, calibración del sistema de control y automatización, vinculadas a una tarjeta mic rocontroladora Nodemcu (ESP-8266).

Palabras claves robótica educativa; áreas STEM; trabajo colaborativo; sistemas de control y automatización.

Abstract Development environments - SDIs - and their applications in measurement-based natural science experiments have been widely welcomed for efficiency and ease of testing scientific theories; By having more interaction with control and automation systems as a pedagogical strategy in the classroom, in secondary educational institutions, it has allowed students, specifically from ninth to eleventh grades, to achieve greater confidence, motivation, collaborative work and use of ICT for the learning of natural sciences, using significant learning for the design and construction of an automated greenhouse for the germination of black mangrove (Avicennia germinans) obtaining optimal growth and conservation of ideal environmental conditions from programming, calibration of the control and automation, linked to a Nodemcu microcontroller card (ESP-8266).

Keywords educational robotics; STEM areas; collaborative work; control and automation systems. 


\section{Introducción}

En la Institución Educativa Soledad Román de Núñez (INEDSOR), desde el año 2010 el área de Ciencias Naturales, ha venido orientando y trabajando en la construcción, sostenimiento y mantenimiento de un vivero escolar para el mejoramiento de la ornamentación institucional y también para contribuir a la protección de cultivos nativos como parte del Proyecto Ambiental Escolar (PRAE). El vivero escolar inició con muchas expectativas, pero en el transcurrir del tiempo se presentaron varios inconvenientes. Se identificó que la falta de mantenimiento por parte de la comunidad educativa afectaba notablemente su sostenimiento, principalmente en los periodos vacacionales. Además de no tener un adecuado control de las condiciones ambientales como temperatura, humedad del suelo, humedad del aire, luminosidad y condiciones fisicoquímicas del suelo, las cuales inciden en el desarrollo de las plantas, tales como el Tomate (Solanum lycopersicum), Maíz (Zea mays) y Ají dulce (Capsicum Annuum), siendo esta la principal planta cultivada en el vivero institucional (Montes, 2017).

En consecuencia, los cultivos no tuvieron un adecuado crecimiento, incluso se producían decesos a causa de la falta de agua, abono y demás cuidados necesarios que requieren las plantas. Por otra parte, las implicaciones económicas y prestaciones sociales que generan contratar un trabajador para esta labor, aumentan los gastos en personal, sin mencionar que este rubro no se encuentra en el presupuesto institucional.

Por lo anterior, desde la enseñanza de las ciencias naturales, específicamente desde la física, y la apropiación de las herramientas de Tecnología de la Información y la Comunicación (TIC), se propone una alternativa de solución fundamentado en un sistema de control y automatización basado en la placa NODEMCU que se alimente de energía solar fotovoltaica para manejar cultivos controlados por sensores, con el propósito de lograr la automatización total de un invernadero, donde el usuario solo participe de forma indirecta para el mantenimiento y cuidado. Esto permitiría una menor inversión de tiempo y un control de variables físicas del cultivo garantizando un buen índice de calidad y productividad en este.

\section{Antecedentes}

En el camino de la búsqueda de información se encontraron investigaciones de gran relevancia para este trabajo de investigación, lo que permitió avanzar teniendo en cuenta:

(a) El funcionamiento de otras placas de hardware, como por ejemplo la Arduino MEGA, los sensores y las variables medidas, la forma de comunicación, lectura, monitoreo y envío de datos (Baltazar, Enciso y Vargas, 2014; Castro, 2013; Mamani, Villalobos y Herrera, 2017; Sepúlveda, 2014);

(b) Las consecuencias del cambio climático sobre las plantas que se cultivan, identificación de las variables (temperatura, humedad del suelo, humedad relativa, luminosidad) a controlar para mantener sus óptimas condiciones;

(c) La viabilidad del sistema de control y automatización a través de la identificación de los sensores y actuadores, leyendo los datos en tiempo real;

(d) El uso de software y hardware libre para bajar los costos del sistema de control y automatización (Baltazar et al., 2014; Fernández, Osorio, Álvarez y Aldana, 2013; Perea, 2016; Mamani et al., 2017);

(e) El rol esencial de los sensores y actuadores para manipular las condiciones óptimas de un cultivo en particular, independientemente de los factores externos;

(f) La opción de emplear energías limpias y renovables para el funcionamiento del sistema de control y automatización, según López de Paz (2016); y también usar a favor de la investigación el Internet de las Cosas (IoT), de tal manera que se pueda realizar en un entorno que facilite la accesibilidad a los componentes, sensores y actuadores, según Mamani et al. (2017). 
Es importante señalar que algunos factores son críticos, desde la perspectiva de la enseñanza y la contribución al aprendizaje colaborativo de las ciencias naturales empleando nuevas estrategias didácticas, aportando al éxito de la investigación, tales como son: a) conocimiento de programación por bloques o código, b) manipulación de componentes eléctricos, c) desarrollo del pensamiento lógico, d) calibración de los sensores y actuadores, e) conectividad a Internet y f) conocimiento de las condiciones del cultivo a sembrar. Pero, sobre todo la motivación por investigar e innovar que se incentiva en los estudiantes.

Todo lo anterior, contribuye a construir sistemas de control y automatización en el aula apropiando una estrategia didáctica de manera innovadora a través de las ciencias y el uso de las TIC. De la tarjeta Arduino se puede describir como una Tarjeta electrónica reprogramable a través de una plataforma de código abierto basada en hardware y software flexibles, que por su fácil uso, se convierte en la opción para personas que no han programado anteriormente en su vida. El microcontrolador de la placa se programa o reprograma en lenguaje Arduino, permitiendo el desarrollo de proyectos que pueden ser autónomos o ser viables para comunicar con software en ejecución en un computador. Su principal característica es la variedad de sensores que se pueden conectar a su placa. Algunas de las ventajas, según Herrador (2009) son:

- Bajo costo: Sus costos son relativamente bajos comparado con otras herramientas para realizar las mismas funciones.

- Compatibilidad de SO: Su ejecución soporta varios sistemas operativos. IDE

- Sencillo: Su entorno de programación es sencillo para principiantes y escalable (flexible) para usuarios avanzados, por su característica de software libre y código abierto.

Código abierto y hardware extensible: Su estructura facilita a inexpertos la construcción de sus propios proyectos de acuerdo a sus necesidades $y$ requerimiento de hardware.

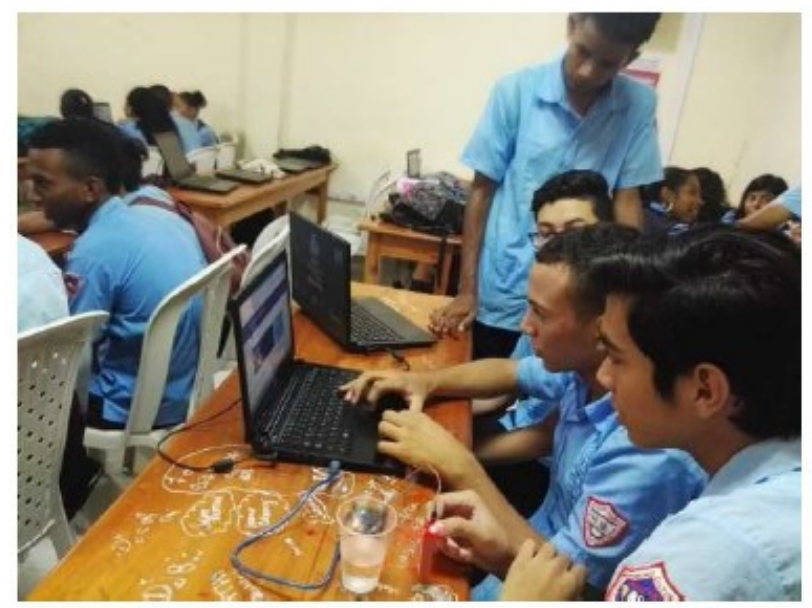

Fig. 1. Diseño de programación y construcción del sistema de control y automatización.

\section{Metodología}

\subsection{Descripción general}

Los sistemas de control y automatización que se emplearon en la Institución Educativa Soledad Román de Núñez, se plantearon desde situaciones problemáticas como lo es en el vivero escolar, evidenciando la necesidad de crear, organizar y acompañar a grupos de investigación que se enfocarán en la problemática.

Lo anterior, motivó a los profesores del área de Ciencias Naturales e Informática a socializar entre los estudiantes (ver Figura 1) la participación en los grupos de investigación del Programa ONDAS de Colciencias, teniendo claridad sobre el objetivo de la investigación que era controlar las condiciones ambientales para la germinación de cultivos nativos y que la metodología empleada sería el trabajo colaborativo entre estudiantes y docentes. Por lo tanto, surgió la necesidad de realizar una búsqueda bibliográfica que permitiera vislumbrar una solución a la situación de medir diversas variables en tiempo real (ver Figura 2 y Figura 3).

En el camino por la investigación, adicionalmente siguiendo la ruta metodológica del Programa ONDAS - COLCIENCIAS, fueron surgiendo varias alternativas para emplear los sistemas de control y automatización, 
aquí se conspiró una estrategia didáctica en la enseñanza de las ciencias naturales en el aula empleando estos nuevos elementos de aprendizajes y enseñanza, conllevando a realizar cambios necesarios, tanto para verificar las condiciones ambientales, aportar los cambios de las variables físicas y nutricionales del mangle negro, a través de una serie de pasos tales como seleccionar e instalar el hardware adecuado al invernadero, diseñar, programar y calibrar el sistema de control para que los intervalos de medida de las magnitudes físicas se mantuvieran (DANE, 2015).

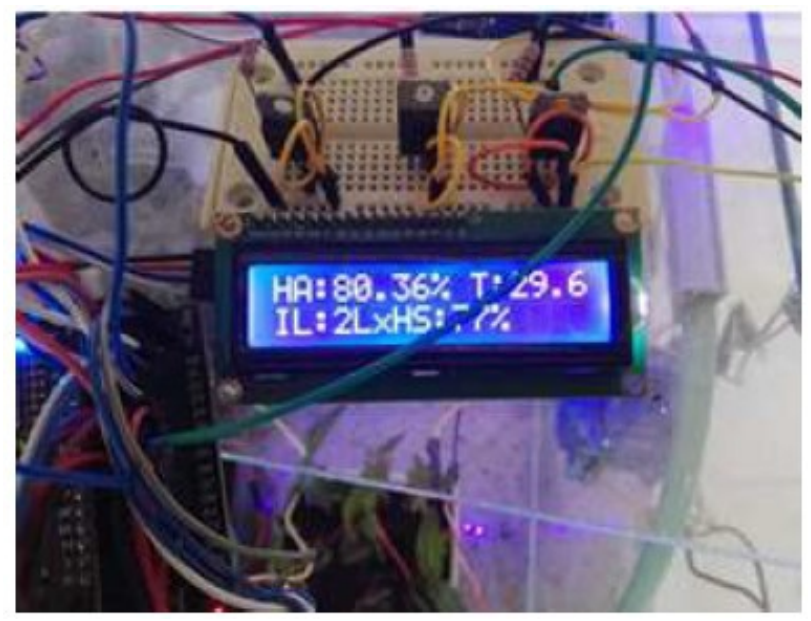

Fig. 2. Sistema de control con los datos de las variables de estudio.

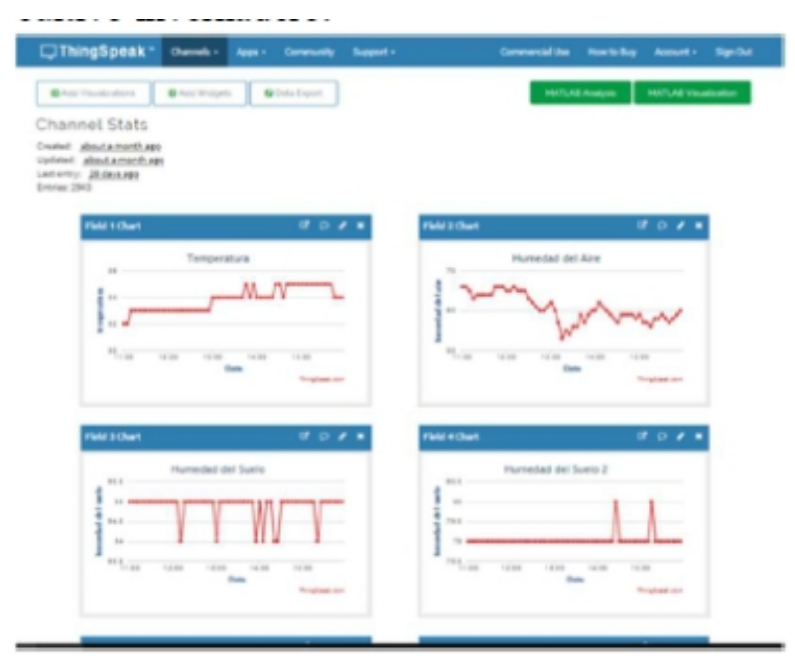

Fig. 3. Gráficos de las variables de estudio en ThingSpeak.
Finalmente, después de realizar un prototipo inicial con la placa Arduino, se decidió implementar la tarjeta NodeMCU, que beneficiaba más la investigación por parte de los estudiantes en cuestiones de costos, ésta última resultó más útil, frente a la Arduino porque presenta ventajas en los costos e incorporación de un microcontrolador Wifi, que permitió a los estudiantes acceder a datos desde la web. Ventaja que se reflejó, además, en el nuevo aprendizaje que adquirieron los estudiantes.

\subsection{Recursos}

(i) Diseño, montaje y calibración del sistema de control: En un trabajo colaborativo: estudiantes, docentes de la Institución Educativa y profesionales interdisciplinares (de diferentes sectores académicos) apoyaron el proceso de investigación, se pudo diseñar el invernadero y el sistema de control, además construir la estructura física del invernadero y su automatización, también realizar la codificación y calibración de sensores, actuadores encargados de la automatización del invernadero dentro de los intervalos permitidos.

(ii) Resultados: Los estudiantes recibieron cátedra de lenguajes de programación, sistemas de control y automatización, desarrollando la programación del sistema invernadero, programación en lenguaje $\mathrm{C}++$ y blocks, desarrollo del pensamiento computacional y lógica matemática. También se realizó experimentación con Arduino UNO, Mega, Leonardo y Tarjeta Nodemcu, sensores de temperatura, humedad de aire, humedad del suelo, intensidad lumínica, presión atmosférica y altura, y adicionalmente emplearon recursos web para la adquisición de datos en tiempo real y por conexiones inalámbricas.

(iii) Materiales: El inicio de la investigación se realizó con Kit de electrónica, placa NodeMCU, Lenguaje de programación $\mathrm{C}++$, computadores, Conectividad a internet, sustratos, semillas de mangle negro, ruta metodológica del programa ONDAS de Colciencias, kit de sensores (humedad del suelo, humedad relativa, temperatura, intensidad lumínica), 
prototipo invernadero, actuadores (bomba de agua, electroválvula, luces RGB (violeta), extractores)

\subsection{Actividades}

Con la estrategia de los sistemas de control se proyecta la búsqueda de los datos, que los sensores pueden captar de las variables de estudio, por lo que inicialmente se desarrolla el pensamiento computacional, la lógica, lenguaje de programación, condicionales y variables con experiencias significativas como juegos de leds, proyectos experimentales y la robótica educativa, en esta última usando sensores de ultrasonido, de infrarrojo y sensor de fuego para cumplir con retos donde se manifiesten habilidades y destrezas en solución de problemas y manejo de las variables. También se recibió la asesoría (ver Figura 3) por parte de la Universidad Tecnológica de Bolívar a través del Programa ONDAS - COLCIENCIAS, que involucra a otras entidades de la región.

\subsection{Escenario de ejecución}

(a) Descripción: La investigación se desarrolló como proyectiva, experimental. En primer lugar, es proyectiva debido a que se propuso un sistema de control y automatización de variables físicas con sensores y actuadores que a partir de la página web permitía la recolección de los datos y verificación de su funcionamiento.

Según Hurtado (2011), la investigación proyectiva consiste en la elaboración de una propuesta o de un modelo, para solucionar problemas o necesidades de tipo práctico o experimental, ya sea de un grupo social, institución, un área en particular del conocimiento, partiendo de un diagnóstico preciso de las necesidades del momento, los procesos explicativos o generadores involucrados y las tendencias futuras. En segundo lugar, experimental por lo que se construyó, calibró y puso en funcionamiento el sistema de control del invernadero donde permitió el estudio las condiciones ambientales ideales y óptimas del crecimiento del cultivo de mangle negro (Avicennia germinans) en la Institución Educativa Soledad Román de Núñez. En cuanto al diseño, se consideró de campo por cuanto en la misma, el fenómeno se observa tal y como se presenta (Sampieri et al., 2014).

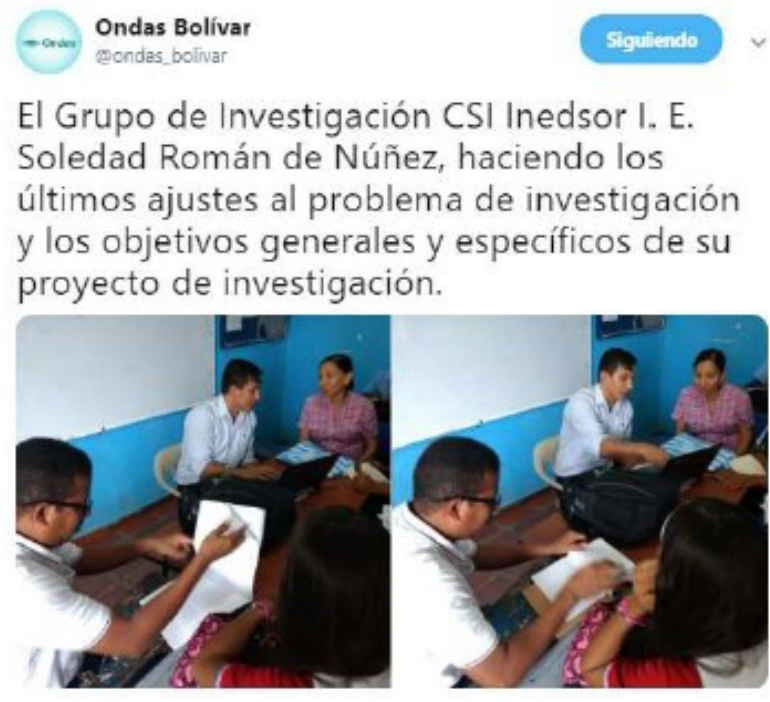

\section{Ondas Bolivar erondas bolivar}

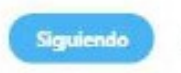

El Grupo de Investigación CSI Inedsor de la Institución Educativa Soledad Román De Nuñez en la revisión de avances en el informe final.

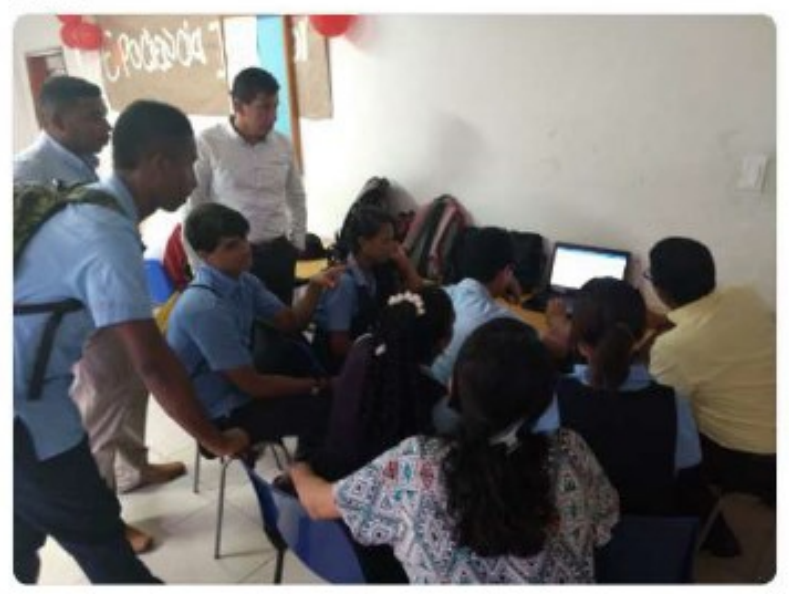

Fig. 4. Asesorías recibidas por parte de la Universidad Tecnológica de Bolívar a través del Programa ONDAS COLCIENCIAS. 
(b) Método e instrumento: Según la perspectiva temporal de recolección de los datos, esta investigación se consideró de diseño transversal, por cuanto la variable objeto de estudio se midió en un solo momento o periodo (Del castillo et al., 2014). De igual manera, el diseño fue experimental, por cuanto la variable se manipula (López-Roldán y Fachelli, 2015). Respecto a la muestra de estudio, estuvo constituida por tres semilleros con diferentes sustratos que en total suman 38 mangles negros sujetos a las mismas condiciones ambientales; está ubicada en el departamento de Bolívar, ciudad de Cartagena.

(c) Instrumentos de la investigación: Como técnica de recolección de información se utilizó una tarjeta Nodemcu con conexión wifi, que, desde los instrumentos de medición directa de sensores, que son dispositivos encargados de transformar los cambios de magnitudes físicas en señales eléctricas, donde los datos se envían a la página web ThingSpeak, un recurso que permite visualizar por gráficos y recolectar los datos de la temperatura, humedad del aire, humedad de suelo, presión atmosférica, intensidad lumínica. Para la construcción del instrumento se usó un sistema de programación y control para el mantenimiento del cultivo de mangle negro, para la temperatura puede oscilar entre los $21^{\circ} \mathrm{C}$ siendo esta la mínima y los $47^{\circ} \mathrm{C}$ la máxima, la humedad relativa oscila entre el $47.13 \%$ la mínima y $72.5 \%$, la máxima según la referencia bibliográfica la cual se ejecutó definiendo sus condiciones ambientales ideales. La validez del instrumento se realizó con la calibración de equipo análogo y de medición manual (PHYWE), los cuales evaluaron el instrumento para permitir que se midiera con exactitud la variable en estudio, para lo cual se constituyó un formato de validación que permitió medir la validez de las condiciones ideales de la planta. El resultado observado en la figura 5 demuestra que fue significativo el crecimiento del mangle negro en los tiempos referenciados en la bibliografía.

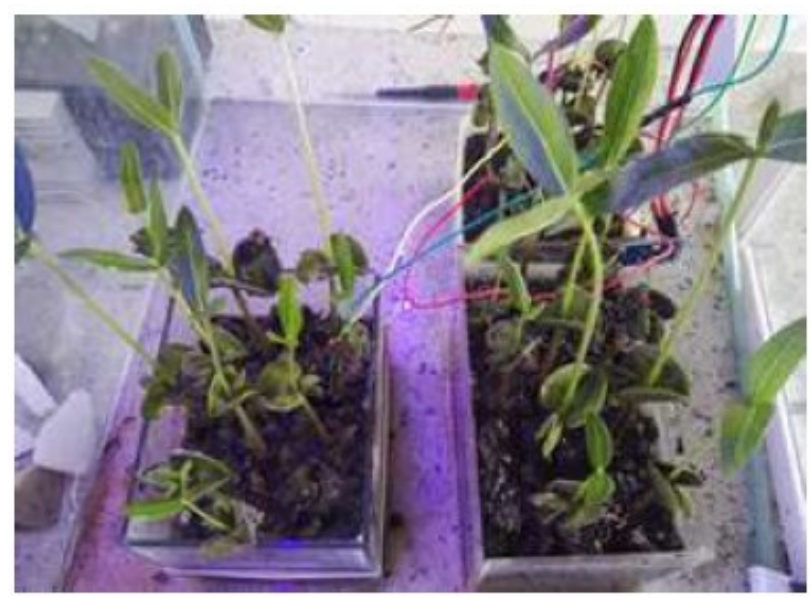

Fig. 5. Crecimiento del Mangle Negro (Avicennia Germinans) en el invernadero.

\section{Resultados}

Con el apoyo y acompañamiento de la Universidad Tecnológica de Bolívar (UTB) y el Programa ONDAS COLCIENCIAS se desarrolló una investigación que permitió controlar las condiciones ambientales para la germinación del mangle negro en un trabajo colaborativo entre estudiantes, docentes y grupo interdisciplinar de profesionales del sector académico. En la figura 6 y figura 8 se evidencia la participación del grupo de Investigación en el Encuentro Regional, una vez se clasificó en la etapa anterior en el Encuentro Departamental, luego se obtuvo un cupo para llegar al Encuentro Nacional "Yo Amo La Ciencia" del Programa ONDAS - COLCIENCIAS, incluso la consecución de representar a Colombia en la Feria Nacional de.

Para la construcción del invernadero automatizado, se conocieron los diferentes tipos, permitiendo elegir el de tipo capilla, ya que era el que más se acercaba a la solución de las necesidades de espacio y variables físicas de contexto.

Se creó el sistema de control, para medir las variables que afectan su uso con la Nodemcu ESP8266 y sensores que permiten controlar, adquirir datos, realizar comparaciones y ejecutar acciones respecto a la germinación y crecimiento del mangle negro. 


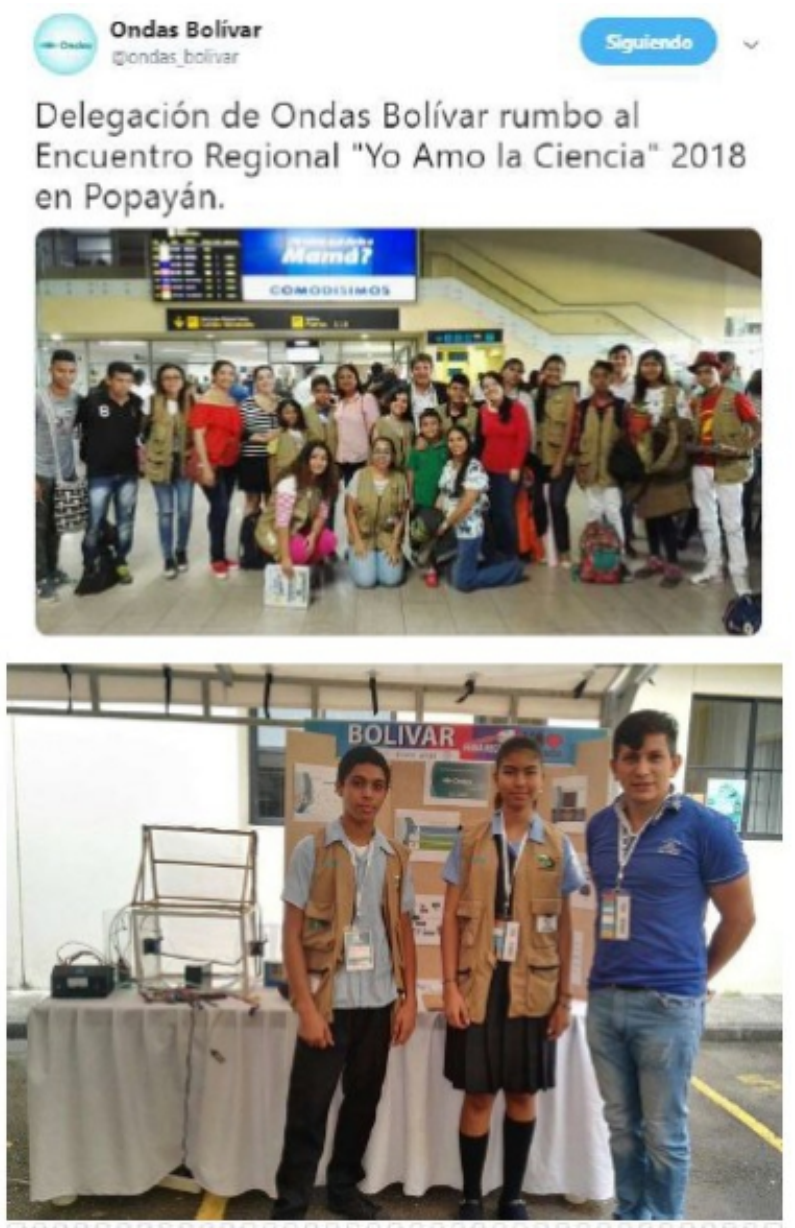

Fig. 6. Participación en los Encuentro Departamental, Regional y Nacional "Yo Amo La Ciencia" del Programa ONDAS - COLCIENCIAS.

Fue posible la adquisición de conocimientos y manejo del lenguaje de programación $\mathrm{C}++$ y Arduino. Gracias a estos conocimientos sobre lenguajes de programación, plataforma IDE Arduino, Atom, mecatrónica, electricidad se pudieron llevar a cabo soluciones de problemas de contexto para el mejoramiento de nuestro entorno escolar, social y ambiental.

Se adquirió experiencia en la instalación eléctrica y electrónica de baja tensión por los conocimientos relacionados con la tarjeta Arduino, la shield, conexiones de circuitos eléctricos, ver Figura 7, planchado de circuitos en baquelita, visualizar comportamiento de la señal de entrada y salida de la
Nodemcu y los tipos de cuidados que se debe tener en el momento de las conexiones eléctricas.

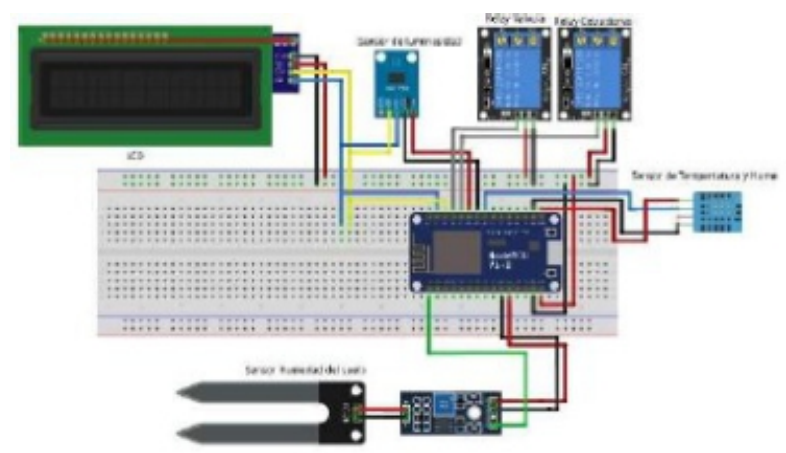

Fig. 7. Diseño del circuito para controlar el cultivo invernadero.

Se logró describir la dependencia del crecimiento de la planta con la humedad relativa de suelo, temperatura del aire, presión atmosférica y el control de variables evolucionando en el tiempo, como son las variables de entrada que tiene el sistema y el control para que la planta de mangle no sufra con respecto a los cambios climáticos externos al invernadero.

\section{Conclusiones y recomendaciones}

Al implementar en la enseñanza de las áreas de ciencias naturales, específicamente física y tecnología, la estrategia didáctica que usa sistemas de control y automatización en el aula, se observó significativamente mayor motivación en el estudiante para la consecución de sus objetivos de aprendizajes.

El rol del profesor incentivó mayor motivación por el uso de las TIC en los estudiantes y facilitó las herramientas en el proceso de enseñanza para generar un trabajo colaborativo entre sus pares, creó espacios que condujeron a retos educativos que se contextualizan en los contenidos curriculares de las áreas llamadas STEAM, ciencias, tecnología, ingeniería, arte y matemáticas.

Por lo anterior, los sistemas de control y automatización en el aula, permiten implementar una estrategia didáctica en la enseñanza de las ciencias, 
para desarrollar competencias tecnológicas en los estudiantes. De forma transversal fortalece las habilidades y destrezas del rol del estudiante como investigador y constructor de su propio aprendizaje, con la comprobación y la construcción de los sistemas de control: en proyectos experimentales del área de las ciencias naturales, casos experimentales, como demostración de teorías y leyes de ciencias físicas, estudio de la germinación del mangle negro en el invernadero, obteniendo eficiencia en su crecimiento en los primeros días.

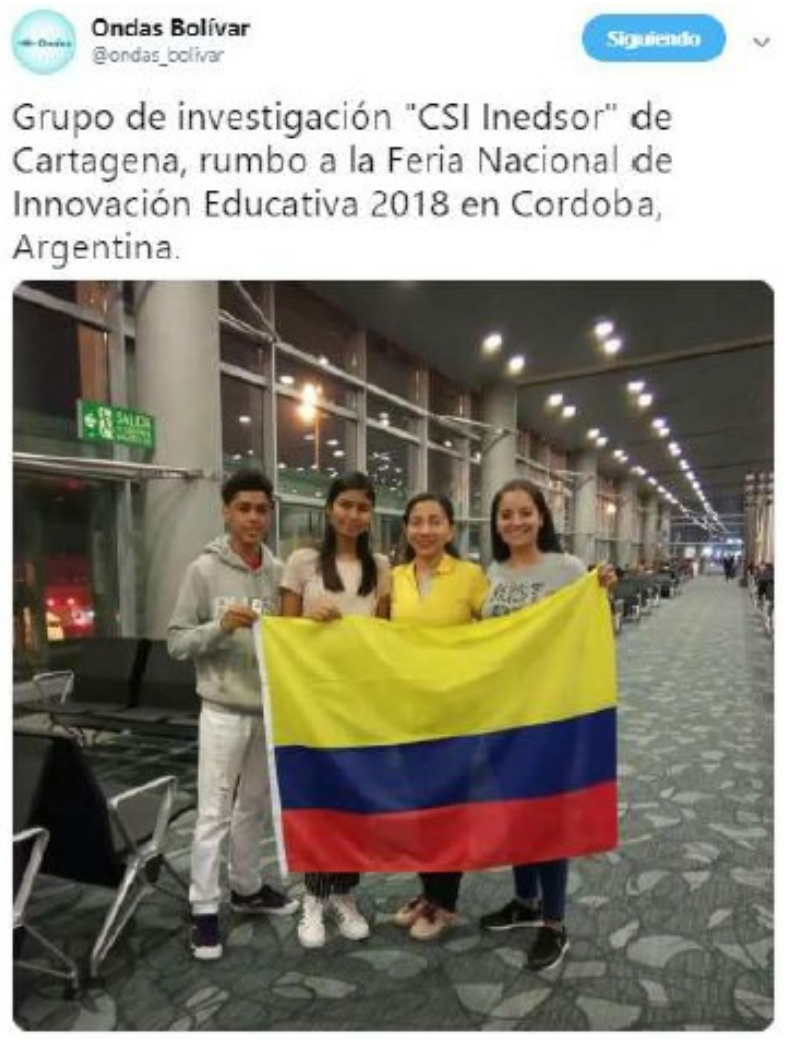

Fig. 8. Participación a nivel Internacional en la Feria Nacional de Innovación Educativa en Argentina.

Adicionalmente, el rol del profesor como orientador del proceso de enseñanza, encarrila el aporte del estudiante mediante la experimentación y sin demeritar la importancia que tiene la fundamentación teórica de las ciencias. La experiencia demostró que se vuelve entonces atractiva la investigación con componentes tecnológicos, que, mediante la manipulación de sistemas de control y automatización, puedan llevar a cabo otros proyectos institucionales, y hasta de ciudad, identificando la situación problemática e implementando los sistemas de control y automatización en las aulas de clases.

\section{Agradecimientos}

Universidad de Cartagena, Universidad Tecnológica de Bolívar, Fundación Universitaria Tecnológico de Comfenalco, Programa ONDAS de COLCIENCIAS, Institución Educativa Soledad Román de Núñez.

\section{Referencias}

Baltazar Aguilar, J., Enciso Hernández, D., \& Vargas Domínguez, M. A. (2014). Diseño e Implementación de un dispositivo digital para el control de la temperatura en un invernadero de tomate. México D.F.: Instituto Politécnico Nacional.

Castro Domínguez, A. (2013). Sistema de control de temperatura a través de Arduino y la tecnología GPRS/GSM. //Castro Domínguez, Alberto (2013). Sistema de control de temperatura a través de Arduino y la tecnología GPRS/GSM. Proyecto Fin de Carrera / Trabajo Fin de Grado, E.U.I.T. Telecomunicación (UPM) [antigua denominación], Madrid. (http://oa.upm.es/22322/)

DANE. (2015). El cultivo del pimentón (Capsicum annuum L) bajo invernadero. Boletín mensual insumos y factores asociados a la producción agropecuaria, 112.

Del Castillo Cruz, C. (2014). Metodología de la investigación. México: Patria.

Fernández Sánchez, S., Osorio Hernández, E., Álvarez Sánchez, E., \& Aldana Franco, R. (2013). Control de Temperatura de un Invernadero a Escala mediante Programación en Arduino. Congreso Nacional de Control Automático, 413-417.

Herrador, R. E. (2009). Guía de Usuario de Arduino. Universidad de Córdoba (España).

Hurtado, J. (2015). El Proyecto de Investigación. Comprensión holística de la metodología y la investigación. Sexta Edición. Caracas: Ediciones Quirón.

López de Paz, R. G. (2016). Diseño del sistema de control de temperatura de un invernadero. Lima: Pontificia Universidad Católica del Perú. 
Melo Jaimes, E., Payares Benítez, D. M., \& Baldiris Navarro, S. M. (2019). Sistemas de control y automatización en el aula: Estrategia didáctica en la enseñanza de las ciencias. Innovación, tecnología y creatividad. Teknos Revista Científica, 19(2), 20-28.

López Roldan, P., \& Fachelli, S. (2015). Metodología de la investigación social cuantitativa. https://ddd.uab.cat/record/129382, 20-24.

Mamani, M., Villalobos, M., \& Herrera, R. (2017). Sistema web de bajo costo para monitorear y controlar un invernadero agrícola. Ingeniare. Revista chilena de ingeniería, 25(4), 599- 618.

Montes Hernández, A. F. (2017). El ají dulce (Capsicum annuum) como alternativa de producción agrícola sostenible en el corregimiento de Caracol-Toluviejo, departamento de Sucre.

Perea Palacios, J. W. (2016). Diseño de un sistema de monitoreo, registro $y$ control de temperatura $y$ humedad para un cultivo de invernadero. Pereira: Universidad Tecnológica de Pereira.

Sampieri, R. H., Collado, C. F., Lucio, P. B., Valencia, S. M., \& Torres, C. P. M. (1998). Metodología de la investigación (Vol. 6). México, DF: Mcgraw-hill.

Sepúlveda, E. J. G. (2014). Control de temperatura y humedad relativa para un deshidratador solar de frutas (Doctoral dissertation, Universidad Tecnológica de Pereira. Facultad de Tecnologías. Tecnología Mecánica). 